\title{
A PRÁTICA DA ATIVIDADE FÍSICA EM MULHERES SUBMETIDAS À CIRURGIA POR CÂNCER DE MAMA: PERCEPÇÃO DE BARREIRAS E BENEFÍCIOS
}

\author{
Maria Antonieta Spinoso Prado ${ }^{2}$ \\ Marli Villela Mamede ${ }^{3}$ \\ Ana Maria de Almeida ${ }^{4}$ \\ Maria José Clapis ${ }^{4}$
}

Prado MAS, Mamede MV, Almeida AM, Clapis MJ. A prática da atividade física em mulheres submetidas à cirurgia por câncer de mama: percepção de barreiras e benefícios. Rev Latino-am Enfermagem 2004 maio-junho; 12(3):494-502.

O exercício físico pós-mastectomia é importante para prevenção da limitação articular, linfedema, alterações posturais, fibrose muscular ou aderência tecidual da área cirúrgica. Objetivo: identificar as crenças a respeito da prática da atividade física, a percepção sobre os benefícios e barreiras à sua realização por um grupo de mulheres submetidas à cirurgia por câncer de mama, segundo o Modelo de Crenças em Saúde. Amostra: 30 mulheres atendidas num serviço especializado em reabilitação de mastectomizadas. Procedimento: aplicou-se um questionário contendo questões relacionadas ao conhecimento e percepções sobre a prática da atividade física, bem como aos benefícios e barreiras à sua realização. Conclusões: o grupo estudado percebeu um conjunto de benefícios sendo a falta de força de vontade a barreira mais mencionada, o conhecimento sobre a importância e a necessidade dos exercícios físicos com a presença de um profissional e o suporte dos familiares foram os incentivos mais referidos pelas mulheres estudadas.

DESCRITORES: neoplasias mamárias; atividade motora; reabilitação, enfermagem

\section{PHYSICAL TRAINING PRACTICE IN WOMEN SUBMITTED TO BREAST CANCER SURGERY: PERCEPTION OF BARRIERS AND BENEFITS}

The importance of post-mastectomy physical training lies in the prevention of joint limitation, lymphedema, postural alterations, muscle fibrosis or tissue adherence to the surgical area. Objective: to identify beliefs on physical training practice, perceptions of barriers and benefits in a group of mastectomized women, in accordance with the Health Beliefs Model. Sample: 30 women assisted at a specialized rehabilitation service for mastectomized women. Procedure: a questionnaire was applied with questions concerning knowledge and perceptions on physical training practice, as well as its benefits and barriers. Conclusions: the studied group perceived a set of benefits, while the lack of willpower was the most mentioned obstacle; knowledge on the importance and need for physical training with the presence of a professional and family support were the most mentioned incentives.

DESCRIPTORS: breast neoplasms; motor activity; rehabilitation; nursing

\section{LA PRÁCTICA DE ACTIVIDAD FÍSICA EN MUJ ERES SOMETIDAS A CIRURGIA POR CÁNCER DE MAMA: PERCEPCIÓN DE BARRERAS Y BENEFICIOS}

La importancia del ejercicio físico post-mastectomía está en la prevención de la limitación articular, linfedema, alteraciones posturales, fibrosis muscular o adherencia del tejido del área quirúrgico. Objetivo: identificar las creencias y percepciones sobre los beneficios y barreras a la práctica de la actividad física por un grupo de mujeres mastectomizadas, de acuerdo con el modelo de creencias en salud. Muestra: 30 mujeres atendidas en un servicio especializado en rehabilitación de mastectomizadas. Procedimiento: fue aplicado un cuestionario con cuestiones relacionadas al conocimiento y percepciones sobre la práctica de la actividad física bien como a los beneficios y barreras para su realización. Conclusiones: el grupo estudiado se dio cuenta de un conjunto de beneficios, y mencionó la falta de ganas como la mayor barrera. El conocimiento sobre la importancia y la necesidad de los ejercicios físicos con la presencia de un profesional y el apoyo de los familiares fueron los incentivos más referidos por las mujeres estudiadas.

DESCRIPTORES: neoplasmas de la mama; actividad motora; rehabilitación; enfermería

\footnotetext{
${ }^{1}$ Trabalho extraído da dissertação de mestrado do programa de Enfermagem em Saúde Pública da Escola de Enfermagem de Ribeirão Preto, da Universidade de São Paulo. Projeto subvencionado pelo Ministério da Saúde - Secretaria de Assistência à Saúde/Secretaria Executiva Projeto REFORSUS; ${ }^{2}$ Mestranda do Programa de Enfermagem em Saúde Pública, e-mail:msprado@eerp.usp.br; ${ }^{3}$ Professor Titular; ${ }^{4}$ Professor Doutor. Escola de Enfermagem de Ribeirão Preto, da Universidade de São Paulo, Centro Colaborador da OMS para o desenvolvimento da pesquisa em enfermagem
} 
A mastectomia, sobretudo acompanhada da radioterapia, pode determinar complicações físicas, imediata ou tardiamente à cirurgia, tais como: limitação e diminuição de movimentos de ombro e braço, linfedema e variados graus de fibrose da articulação escapuloumeral ${ }^{(1-}$ 2).

A prática de exercícios físicos relacionados com a reabilitação pós-mastectomia, bem como a orientação destes, são intervenções importantes na assistência pósoperatória à mulher pois têm como finalidade prevenir ou minimizar o linfedema ou perda de mobilidade no ombro ${ }^{(2)}$.

A reeducação da cintura escapular e do membro superior é uma necessidade básica para a paciente submetida à cirurgia por câncer de mama, seja qual for a técnica empregada. Seu objetivo principal é restabelecer o mais rapidamente possível a função do membro superior, além de atuar como fator preventivo à formação de cicatriz hipertrófica, aderências e linfedema de membro superior ${ }^{(3)}$.

Em nossa experiência profissional, junto a um grupo que presta assistência integral à mulher mastectomizada, observamos que a grande maioria das mulheres não incorpora a prática regular de exercícios físicos mesmo sabendo da possibilidade de complicações para o resto de suas vidas. Observamos, também, a falta de adesão à sua pratica, mesmo entre mulheres que relatam que, ao praticá-lo, sentem-se bem, pois os movimentos do braço melhoram chegando até à diminuição do linfedema.

Da mesma forma, ao testar um protocolo para tratamento do linfedema pós-cirurgia por câncer de mama, que incluía exercícios físicos, constatou-se que um grupo de mulheres estudadas apresentou dificuldades de aderência à realização rotineira dos exercícios físicos ${ }^{(4)}$.

Assim, entendemos que a equipe de saúde exerce um papel importante na prevenção, profilaxia e tratamento de tais complicações, no entanto, a prática de atividades relacionadas à reabilitação física de mulheres pós-cirurgia por câncer de mama tem sido um grande desafio.

\section{OBJETIVO}

O objetivo deste estudo foi identificar as crenças a respeito da prática da atividade física, a percepção sobre os benefícios e barreiras à sua realização por um grupo de mulheres submetidas à cirurgia por câncer de mama.
Esta pesquisa foi desenvolvida com um grupo de mulheres mastectomizadas que freqüentaram, no período de março a agosto de 2001, o Núcleo de Ensino Pesquisa e Assistência na Reabilitação de Mastectomizada-REMA, sediado na Escola de Enfermagem de Ribeirão Preto/USP. O núcleo oferece atendimento três vezes por semana, o qual consta de um programa de atividades visando à recuperação física, emocional e social.

A assistência prestada, com vistas à reabilitação física, dentre seus protocolos, consta de um programa de exercícios físicos individuais, em aparelhos e em grupo, ocasião em que é enfatizada a importância da prevenção do linfedema, pois, uma vez instalado, torna-se difícil sua regressão total.

Sujeitos do estudo

Até agosto de 2001, o REMA contava com 545 mulheres cadastradas, sendo que 92 destas compareceram ao serviço no período estudado e participaram das etapas propostas para a reabilitação física e/ou emocional.

O processo de recrutamento dos sujeitos ocorreu por meio de convite, sendo informadas sobre o objetivo da pesquisa e a forma de sua participação. Os critérios de inclusão foram: mulheres submetidas à cirurgia por câncer de mama, que freqüentassem o núcleo no período de março a agosto de 2001, terem de 3 meses a dois anos póscirurgia; após os esclarecimentos necessários, terem lido e assinado o Consentimento Livre e Esclarecido. Do total das 92 mulheres, 30 atenderam aos critérios de seleção e constituíram a amostra do estudo.

O presente projeto obteve aprovação pelo Comitê de Ética em Pesquisa, da EERP/USP.

Procedimentos de coleta e análise dos dados

A coleta de dados ocorreu por meio de entrevista individual, com ajuda de instrumento construído para esse fim, com base na literatura sobre a prática de exercícios físicos e nas experiências de profissionais que trabalham com a reabilitação de mulheres mastectomizadas.

O instrumento continha dados de identificação, questões fechadas, mas com abertura de possibilidade 
para justificativa da resposta por meio das quais buscou identificar as crenças sobre a prática de exercícios físicos, as dificuldades e as facilidades percebidas pelas entrevistadas sobre tal prática, a sua importância, assim como o período de realização dos exercícios.

O instrumento foi construído com base no Modelo de Crenças em Saúde, visto que ele busca explicar o comportamento humano quanto ao processo saúdedoença. Esse modelo surgiu na tentativa de explicar por que as pessoas não se previnem a contento contra certas doenças ou não utilizam programas de "screening" para detecção precoce da doença assintomática, como tuberculose, câncer cervical, doenças dentárias, febre reumática, pólio e influenza, serviços estes oferecidos gratuitamente à população(5).

O Modelo de Crenças em Saúde parte do pressuposto de que, para o indivíduo emitir comportamentos preventivos em relação a uma dada doença, necessita acreditar que ele é pessoalmente suscetível àquela doença e que a sua ocorrência deverá ter, pelo menos, moderada seriedade em algum componente de sua vida. E, para realizar ações preventivas ou modificar comportamentos, o indivíduo necessita acreditar nos benefícios de tais mudanças, ou seja, que elas são capazes de reduzir sua suscetibilidade àquela doença ou reduzir a sua seriedade. Segundo esse modelo, há barreiras psicológicas importantes, capazes de impedir que o indivíduo tome ações relacionadas à prevenção de doenças.

Basicamente, o Modelo de Crenças em Saúde lida com quatro variáveis: susceptibilidade, seriedade, benefícios e barreiras percebidas, apesar de haver outras variáveis que podem afetar as percepções individuais, influenciando indiretamente a ação final. Na construção do instrumento, foram elaboradas questões que se dirigiam à identificação de benefícios e barreiras para a prática da atividade física.

Para a análise dos dados, respaldada no Modelo de Crenças em Saúde, agrupamos os conteúdos das justificativas das repostas em itens, a fim de facilitar a sua codificação. Recortes dessas justificativas foram utilizados para melhor compreensão da percepção dos sujeitos sobre a prática da atividade física. Armazenamos todos os dados em um banco, analisando-os quantitativamente, por meio de freqüência.

\section{RESULTADOS E DISCUSSÃO}

\section{Caracterização da amostra}

A idade média das 30 mulheres estudadas foi de 52,7 anos, com variação entre 36 e 69 anos, estando $43,3 \%$ delas na faixa etária de 41 a 50 anos. Quanto ao estado civil, 19 (63,3\%) eram casadas; seis (20\%), solteiras, três (10\%), viúvas e duas (6,7\%), separadas.

Em relação ao grau de instrução, 3 (10\%) eram analfabetas, $9(30 \%)$ tinham menos que oito anos de estudo, 10 (33,3\%), de oito a 11 anos de estudo e 8 (26,6\%), mais que 11 anos de estudo, ou seja, o terceiro grau incompleto ou completo. Quanto à ocupação, 18 $(60 \%)$ eram donas de casa.

Verificamos que, dentre as mulheres submetidas a tratamento cirúrgico, 19 (63,3\%) fizeram cirurgia conservadora, sendo que 9 destas foram submetidas à quadrantectomia, e 10, à nodulectomia. Esse dado revela que a tendência atual de tratamento do câncer de mama tem sido a de favorecer uma melhor qualidade de vida, pois o tratamento mais conservador pode contribuir para uma melhora na aceitação da imagem corporal ${ }^{(6)}$.

Em relação ao tempo de cirurgia, no momento da entrevista, a maioria delas, 20 (66,7\%), estava no primeiro ano de pós-operatório, ou seja, 11 (36,7\%) tinham de três a seis meses pós-cirurgia e 9 (30\%), de 7 a 12 meses de pós-cirurgia. Esses períodos são de grande importância para o enfrentamento das dificuldades decorrentes do procedimento cirúrgico, pois é no decorrer deles que as mulheres encontram maior dificuldade para alcançar toda a amplitude de movimento do braço do lado operado como também para incorporar procedimentos de autocuidado e prevenção de linfedema desse braço.

Em relação às outras modalidades de tratamento, $22(73,3 \%)$ mulheres tinham sido submetidas à radioterapia, e 5 (16,6\%) estavam com o tratamento em curso. Quanto à quimioterapia, 17 (56,7\%) mulheres foram submetidas a esse tratamento, e 9 (30\%) delas estavam com o tratamento em curso.

O diagnóstico de linfedema é estabelecido quando a mulher apresenta edema com uma diferença entre as medidas dos braços de 1 a 1,5 centímetros; no entanto, quando essa diferença é de até 3 centímetros, o linfedema 
é considerado leve e, de 3 a 5 centímetros, linfedema moderado, acima de 5 centímetros, linfedema severo ${ }^{(7)}$.

Das mulheres estudadas, 14 (46,6\%) apresentavam linfedema leve, $2(6,6 \%)$ com grau moderado, 4 (13,3\%), grau severo, e 10 (33,3\%) não apresentavam tal complicação. Esses dados revelam a importância da prática do exercício físico para profilaxia e prevenção do linfedema.

Conhecimento relacionado à prática do exercício físico

O conhecimento sobre a percepção dos benefícios e barreiras a respeito da prática da atividade física pode ser um importante indicador para o desenvolvimento de programas de reabilitação de pacientes em risco de apresentar limitações físicas, como é o caso de mulheres mastectomizadas. Isso porque a prática da atividade física é um hábito difícil de incorporação, e a consciência da relação atividade física e saúde leva à necessidade de prática regular, especialmente entre indivíduos com doenças crônico-degenerativas ${ }^{(8)}$.

No presente estudo, ao identificarmos as crenças que as mulheres têm a respeito da prática da atividade física, verificamos que $100 \%$ delas identificam-na como boa para a saúde, pois, segundo 19 (63\%) delas, tal prática é capaz de "promover a saúde e prevenir doenças"; 12 (40\%) associam-na como "melhora a disposição", e 6 $(20 \%)$ referiram que "previne o linfedema".

A prática de exercícios físicos, associada ao tratamento do câncer, é descrita como benéfica, pois tem um efeito psicológico positivo no humor, melhora a capacidade funcional, aumenta o apetite e melhora a qualidade de vida dos pacientes ${ }^{(9)}$.

\section{Percepção dos benefícios da atividade física}

Pelas respostas das mulheres quanto aos indicadores de benefícios da prática da atividade física, todas identificaram benefícios tanto relacionados na esfera física quanto na emocional (Tabela 1). Do ponto de vista físico, relataram que "melhoram a disposição", 30 (100\%), que são "bons para as articulações", 30 (100\%), "melhoram os movimentos dos braços e ombros", 30 (100\%), "previne o linfedema”, 28 (93,3\%), e fortalecem os músculos, 27 $(90 \%)$
Tabela 1 - Distribuição das justificativas sobre a percepção de benefícios da prática da atividade física pelas mulheres de um núcleo de reabilitação de mastectomizadas. Ribeirão Preto, 2001

\begin{tabular}{|c|c|c|c|}
\hline Indicador & Justificativas & $\mathrm{N}$ & $\%$ \\
\hline \multirow{5}{*}{$\begin{array}{l}\text { Disposição, } \\
\text { energia, ânimo e } \\
\text { bem- estar } \\
\mathrm{N}=30\end{array}$} & Melhora o corpo & 25 & 83,3 \\
\hline & Melhora a mente & 18 & 60,0 \\
\hline & É prazeroso & 2 & 6,7 \\
\hline & $\begin{array}{l}\text { Pensa em si próprio (auto } \\
\text { estima/imagem corporal) }\end{array}$ & 2 & 6,7 \\
\hline & Outros & 1 & 3,3 \\
\hline \multirow{4}{*}{$\begin{array}{l}\text { Bom para } \\
\text { articulações e } \\
\text { melhora } \\
\text { movimentos dos } \\
\text { braços e ombros } \\
\mathrm{N}=30\end{array}$} & Melhora progressiva & 17 & 56,7 \\
\hline & Trabalha os músculos/corpo & 7 & 23,3 \\
\hline & Evita sinais e sintomas & 5 & 16,7 \\
\hline & Melhora a circulação & 4 & 13,3 \\
\hline $\begin{array}{l}\text { Previne o } \\
\text { linfedema }\end{array}$ & $\begin{array}{l}\text { Movimenta o braço/ } \\
\text { circulação }\end{array}$ & 18 & 60,0 \\
\hline \multirow[t]{4}{*}{$\mathrm{N}=28$} & Sente melhora & 5 & 16,7 \\
\hline & Braço fica mais leve & 2 & 6,7 \\
\hline & $\begin{array}{l}\text { Experiência do REMA/ouve } \\
\text { falar }\end{array}$ & 2 & 6,7 \\
\hline & Outras & 1 & 3,3 \\
\hline \multirow{4}{*}{$\begin{array}{l}\text { Fortalece os } \\
\text { músculos } \\
\text { N=27 }\end{array}$} & Mexe com o corpo & 12 & 40,0 \\
\hline & $\begin{array}{l}\text { Músculo sem estímulo, } \\
\text { atrofia }\end{array}$ & 10 & 33,3 \\
\hline & Depende do exercício & 3 & 10,0 \\
\hline & Não sabe explicar & 3 & 10,0 \\
\hline \multirow{5}{*}{$\begin{array}{l}\text { Evita o estresse, } \\
\text { ajuda a saúde } \\
\text { mental } \\
\mathrm{N}=30\end{array}$} & Relaxa o corpo & 10 & 33,3 \\
\hline & Distração/alívio de tensão & 10 & 33,3 \\
\hline & Pensar em si próprio & 2 & 6,7 \\
\hline & $\begin{array}{l}\text { Disposição/melhora o corpo } \\
\text { e a mente }\end{array}$ & 5 & 16,7 \\
\hline & Se for prática rotineira & 3 & 10,0 \\
\hline Dormir melhor & Relaxa o corpo & 11 & 36,6 \\
\hline \multirow[t]{4}{*}{$\mathrm{N}=28$} & Experiência própria & 2 & 6,6 \\
\hline & Gasta energia & 3 & 10,0 \\
\hline & Melhora mente e corpo & 11 & 36,6 \\
\hline & Indiferente & 1 & 3,3 \\
\hline É prazerosa a & Bom para o corpo & 7 & 23,3 \\
\hline prática da & Bom para a mente & 13 & 43,4 \\
\hline $\begin{array}{l}\text { atividade física } \\
\mathrm{N}=27\end{array}$ & Sente bem & 11 & 36,7 \\
\hline
\end{tabular}

Ao expressarem que a atividade física "melhora a disposição, o ânimo, energia e bem-estar", elas explicam que a própria natureza corporal humana demanda necessidades, aqui traduzidas por elas como benefícios:

Não fomos feitas para sermos estáticas, mas dinâmicas, temos necessidades físicas, orgânicas e psicológicas, de movimentar, participar, comunicar, e o exercício físico, além de proporcionar uma aparência mais agradável, nos dá uma sensação de bem-estar.

A identificação dos exercícios como benéficos não somente para o bem-estar geral, mas também para as articulações, musculatura e movimentação é concordante com os dados de pesquisas quando concluem que o exercício físico, além de manter e melhorar a força muscular, a saúde e a energia, deve ser usado como estratégia para aqueles pacientes oncológicos que relatam fadiga $^{(10-11)}$.

Na mastectomia, o ombro é a articulação mais 
comumente prejudicada devido à imobilização prolongada, e este é um dos fatores responsáveis pelo desenvolvimento de isquemia dos tecidos internos, retenção de metabólitos e edema, apressando, assim, o desenvolvimento de fibrose. A literatura vem apontando que os exercícios ativos são importantes recursos para prevenção dos efeitos deletérios da imobilização ${ }^{(12)}$.

Interessante observar que um grande contingente das mulheres entrevistadas percebe a prática da atividade física como benéfica à prevenção e tratamento do linfedema, pois $28(93,3 \%)$ delas assim o afirmaram. Dessas mulheres, 18 (60\%) justificaram que o movimento do braço melhora a circulação, o que vem ao encontro da literatura quando esclarece que o objetivo do exercício não é somente restabelecer a função articular, mas também estimular a circulação venosa e linfática ${ }^{(3)}$. Ao mesmo tempo, algumas mulheres justificaram que a prática previne o linfedema, quando referiram que sentem melhora do braço $5(16,7 \%)$, e ele se torna mais leve $2(6,7 \%)$.

As mulheres estudadas, ao identificarem benefícios da atividade física relacionados à dimensão emocional, dizem que ela "melhora a disposição, ânimo, energia e bem-estar" - 30 (100\%), que "evita estress, ajuda a saúde mental" - 30 (100\%) e faz com que elas "durmam melhor" - 28 (100\%), além de "sentirem prazer na sua realização" - 27 (90\%).

A prática regular da atividade física traz melhora à saúde psicológica, no bem-estar geral e favorece uma visão positiva de futuro ${ }^{(10)}$.

Neste estudo, todas as mulheres referiram a atividade física como um recurso para evitar o estresse e ajudar na saúde mental, pois acreditam que ela relaxa o corpo 10 (33,3\%), por ser um momento de distração e alívio de tensão - 10 (33,3\%).

A percepção de que a atividade física traz benefícios psicológicos é um importante indicador para minimizar os efeitos adversos ao diagnóstico e tratamento do câncer de mama, pois, segundo a literatura, eles podem levar a estresse prolongado, gerando ansiedade, depressão, insônia, isolamento social, medo da morte e do retorno da doença, influindo na qualidade de vida ${ }^{(13-14)}$.

O linfedema, como uma complicação que pode ser desenvolvida depois da cirurgia de câncer de mama, chega a preocupar mais do que a própria doença, o câncer, ou a mutilação do corpo pela retirada cirúrgica da mama. Ele provoca mudança de hábitos, leva a dúvidas e dificuldades de diversas ordens, assim como alterações emocionais, caracterizando-se como um problema estigmatizante $^{(15)}$.

As justificativas do indicador dorme melhor, como benefício da atividade física, foram as seguintes: $36,7 \%$ das mulheres disseram que conseguiam dormir melhor porque sentiam o corpo relaxado, e outras $36,7 \%$ acreditavam que isso acontecia porque tal prática melhorava a mente e o corpo.

Acreditamos que sentir prazer na realização da atividade física é o primeiro passo para que a prática seja incorporada na rotina diária. Ao se reportarem sobre a questão da prática ser prazerosa, 27 (90\%) mulheres perceberam-na como tal. Sendo que 13 mulheres (43,3\%) justificaram que a atividade física é boa para a mente; 11 $(36,7 \%)$ mulheres referiram que é boa para o corpo, e sete $(23,3 \%)$ mulheres relataram que se sentem bem quando a realizam (Tabela 1).

Percepção das barreiras para a prática do exercício físico

Por entendermos que a percepção de barreiras à prática da atividade física entre mulheres submetidas à cirurgia de câncer de mama relaciona-se a um conjunto de razões ou crenças pessoais, procuramos, neste estudo, identificar, na população estudada, as razões pela não adesão a tal prática.

A falta de força de vontade foi o indicador de barreira mais freqüentemente apontado $29(96,7 \%)$ pelas mulheres estudadas (Tabela 2).

Tabela 2 - Distribuição das respostas dadas pelas mulheres que freqüentavam um Núcleo de Reabilitação de Mastectomizadas sobre as percepções das barreiras ao exercício físico. Ribeirão Preto, 2001

\begin{tabular}{clrr}
\hline Indicador & \multicolumn{1}{c}{ Justificativas } & No & $\%$ \\
\hline Força de & Tirar tempo para si & 8 & 26,7 \\
vontade & Para ter melhora & 8 & 26,7 \\
N=29 & Falta de ânimo, cansaço físico & 7 & 23,3 \\
& Disciplina/rotina & 5 & 16,7 \\
Cansaço & Falta de condicionamento físico & 6 & 20,0 \\
N=10 & Fazer de forma errada & 2 & 6,7 \\
& Quando está fazendo quimioterapia & 1 & 3,3 \\
& Não sabe & 1 & 3,3 \\
Recurso & Depende de onde fizer & 4 & 13,3 \\
financeiro & Academias são caras/individualizado & 3 & 10,0 \\
$\mathrm{~N}=8$ & Transporte & 1 & 3,3 \\
Esforço físico & Falta de condicionamento físico & 8 & 26,7 \\
$\mathrm{~N}=8$ & & & \\
Tempo & A própria pessoa faz o tempo & 1 & 3,3 \\
$\mathrm{~N}=1$ & & & \\
\hline
\end{tabular}

As mulheres justificam que é necessário ter força de vontade, pois, para a adesão a essa prática, há 
necessidade de dedicarem um tempo para si mesmas e que, sem um espaço para si, a incorporação de novas atividades ficam prejudicadas.

A força de vontade para realizar a atividade física para algumas mulheres - $8(26,7 \%)$ - estava atrelada à percepção da necessidade de melhora de sua condição de saúde, como mostra o seguinte recorte: "pois sem a força, a tendência é para o aconchego total, a acomodação, o sedentarismo, resultando obviamente na doença".

Isso revela que esse atributo é de fundamental importância para promover mudanças comportamentais, uma vez que ele é dependente de outros fatores, como estado de ânimo, cansaço e disciplina. Portanto, essas revelações chamam a atenção para a necessidade de uma melhor compreensão do atributo "força de vontade" por parte dos profissionais de saúde, de forma a motivá-las e ajudá-las a trazê-lo para uma região mais central, no espaço de vida de cada uma delas.

Por outro lado, as mulheres identificaram o cansaço e o dispêndio de esforço físico como indicadores de barreiras à atividade física - 10 (33,3\%) - e $8(26,6 \%)$, respectivamente, e a maioria delas justificaramnos a falta de condicionamento físico. O cansaço e ou fadiga são queixas comuns entre as pacientes que estão recebendo tratamento quimioterápico e radioterápico para o câncer de mama, e a atividade física pode ser uma das estratégias para alívio da fadiga ${ }^{(16)}$.

Para alguns autores, os exercícios físicos não aumentam o risco de injúrias músculo- esquelético, embora alguns estudos sugiram que o risco é maior somente quando os indivíduos, principalmente adultos mais velhos, estão engajados em atividades físicas de alto impacto, quando comparados com os de atividade física de baixo impacto $^{(10)}$.

Dentre as mulheres que não identificaram tais indicadores de barreira, elas explicaram que essa atividade deve ser feita de acordo com o limite de cada uma delas e de forma a produzir prazer na sua realização. O seguinte recorte expressa tal percepção: "faz pelo prazer, porque vai sentir bem, faz naturalmente".

Outro motivo que poderia ser alegado como barreira para a prática da atividade física relaciona-se aos recursos financeiros para a sua realização. Para 25 $(83,4 \%)$ mulheres, a questão financeira não foi motivo para a falta de adesão à atividade física, pois 17 delas expressaram que têm consciência de que existem locais públicos e serviços de saúde gratuitos para essa prática, além de justificarem que podem realizá-la em suas próprias casas. A caminhada também foi citada como uma estratégia a ser utilizada, pois não reverte em custo e é acessível a todas elas.

As $5(26,7 \%)$ mulheres que citaram a prática dos exercícios físicos como sendo de custo alto, relacionaramna como academias particulares, recebendo orientação individual de um professor.

A falta de tempo tem sido apontada na literatura como indicador de barreira à pratica da atividade física ${ }^{(17)}$, no entanto, em nosso estudo, 29 (96,7\%) mulheres disseram não acreditar nisso, sendo que $20(66,7 \%)$ delas disseram que "o tempo quem faz é a gente".

Esses achados podem indicar que a falta de interesse ou comprometimento, ao invés da falta de tempo para realização da atividade física, pode ser um fator predisponente à não adesão ou inatividade ${ }^{(10)}$.

Motivação para a prática do exercício físico

De acordo com o Modelo de Crenças em Saúde, alguns estímulos podem eliciar o processo de tomada de decisão, podendo ter origem "interna" (por exemplo, um sintoma) ou "externa" (influência da família, dos amigos, dos meios de comunicação, etc.).

A limitação de movimentos da cintura escapular, ombros e braços e, em especial, o edema de braço, têm sido descritos como importantes seqüelas do tratamento do câncer de mama, e o linfedema, como uma grande ameaça para a vida dessas mulheres.

No presente estudo, foi possível verificar que a grande maioria das mulheres estudadas - 28 (93,3\%) percebeu o linfedema como uma ameaça e que poderia vir a desenvolvê-lo, em algum momento de suas vidas, e, como forma de previni-lo, quase a totalidade das mulheres referiu que realizavam atividade física, em especial os exercícios físicos destinados à sua reabilitação física.

Os exercícios físicos propostos e ensinados no serviço especializado foram aqueles relatados os mais freqüentemente por elas 26 (86,6\%) como fazendo parte do cotidiano de seus espaços de vida. A caminhada 10 (33,3\%), bem como outras modalidades de atividade física - 4 (13,3\%) - foram referidas por elas, sendo que aquelas que costumavam fazer caminhada também realizavam os exercícios prescritos para a sua reabilitação física.

O tipo de atividade física praticada pelas mulheres estudadas está muito mais voltado para a reabilitação física 
pós-mastectomia, cujas atividades relacionam-se ao aumento da amplitude dos movimentos dos braços e ombros e prevenção do linfedema. Isso porque 26 (86,7\%) mulheres referiram realizar os exercícios propostos pelo serviço especializado em reabilitação, realizando-os tanto nos dias de atendimento como no domicílio.

Para que a atividade física funcione como um determinante para a promoção da saúde, prevenção e redução de riscos associados a doenças e ou complicações, como imobilidade e linfedema de braço póscirurgia de câncer de mama, essa prática necessita ser realizada de forma regular e constante.

A realização dessa prática de forma sistemática foi identificada em $13(43,3 \%)$ mulheres que referiram exercitarem-se diariamente, outras $10(33,3 \%)$ o faziam pelo menos três vezes por semana, e $5(16,7 \%)$, apenas uma vez por semana. Esses dados revelam que a adesão à atividade física, conforme recomendada pela literatura ${ }^{(10)}$, ou seja, pelo menos duas a três vezes por semana, foi referida pela grande maioria das mulheres estudadas 23 (76,6\%).

No que diz respeito à hora da realização dos exercícios físicos, $50 \%$ das mulheres disseram que dependiam de um tempo disponível, revelando que essa atividade não era prioritária no seu cotidiano. Entre aquelas que mencionaram uma hora marcada para a realização dos exercícios, algumas desenvolviam no serviço especializado para reabilitação ou num horário préestabelecido, quando não tinham compromissos anteriormente assumidos. Esses dados reforçam a idéia de que a prática da atividade física, de um modo geral, para as mulheres estudadas, é introduzida no seu cotidiano, nos intervalos de tempo disponíveis, demonstrando que ela pode deixar de ocupar um espaço central nas suas vidas.

Ao serem questionadas sobre o tempo que dedicavam à atividade física, ele variou de quinze minutos a uma hora, com média de 43,1 minutos (desvio-padrão de 14,2). No entanto, a maioria das mulheres, 21 (70\%), achou que o tempo dispendido não era suficiente, outras $14(46,7 \%)$ alegaram que gostariam de dedicar mais tempo à atividade física, pois ajudava a melhorar o condicionamento físico, porém manifestaram falta de coragem para aumentarem esse tempo.

Quanto aos estímulos e incentivos para tornar a prática da atividade física mais fácil, as mulheres estudadas identificaram um conjunto de razões para sua adesão ou não. Assim, 11 (36,7\%) mulheres referiram que sabiam da importância e da necessidade dos exercícios físicos para as suas vidas, como é relatado: "a vontade de ficar boa, de ter os movimentos livres", "saber que vai ficar cada vez melhor com o braço", era uma grande motivação para a prática.

Outros indicadores citados como facilitadores ou motivadores para a adesão à prática da atividade física foram: presença de um profissional em grupos especializados para reabilitação, suporte de familiares, uso de música concomitante aos exercícios e reforço das orientações por meio de um manual.

A família (marido, filhos e irmãos) foi o indicador de incentivo para a realização da prática dos exercícios mais freqüentemente relatado, visto que $18(60 \%)$ das mulheres citaram que recebem tanto apoio da família que, em alguns momentos, funcionou de forma negativa, como relatado: "o estímulo é tanto que em alguns casos o apoio já virou cobrança".

Ajudar a família a reconhecer o impacto do câncer de mama é uma tarefa em que a enfermagem pode atuar, pois, após esse impacto, poderá provocar uma perturbação na sua dinâmica, necessitando mudanças e uma nova organização familiar, devido aos desajustes que o câncer de mama pode acarretar ${ }^{(18)}$.

Tais dados representam importantes indicativos para os profissionais de saúde proporem estratégias que possam favorecer uma melhor adesão à pratica das atividades físicas que venham promover um estado de saúde mais equilibrado.

Os programas de atividades físicas destinados a atrair mulheres pós-cirurgia de câncer de mama deveriam incluir mensagens que desfizessem mitos em relação aos exercícios físicos e oferecer oportunidades para vários tipos de atividades que fizessem as mulheres terem prazer $^{(10)}$.

\section{DISCUSSÃo}

A análise dos dados aqui apresentados permitiu perceber que as mulheres identificaram mais benefícios do que barreiras à prática da atividade física. Deixam em evidência que os benefícios percebidos estão associados tanto ao corpo como à mente, pois, para elas, a prática da atividade física traz importantes contribuições para a saúde e prevenção de doenças, além de auxiliar no retorno da funcionalidade dos braços e ombros homolaterais à cirurgia.

A percepção de que a adesão à atividade física 
repercute na dimensão psicológica do ser humano fica evidente quando expressam que esta é uma forma encontrada por elas para relaxamento, distração e alívio de tensão e para alcançar o bem-estar.

De um modo geral, as barreiras percebidas pelas mulheres para a prática da atividade física foram relacionadas à falta de ânimo e condicionamento físico, como também pela própria limitação física. As mulheres dão indicativos de que necessitam ter um tempo para si, sair do comodismo, e, principalmente, terem força de vontade, revelando que estes são qualificadores a serem conquistados por elas mesmas

Analisando os dados referentes às respostas das mulheres sobre conhecimento e percepção a respeito das repercussões da atividade física para a sua saúde, foi possível identificar que aspectos positivos dessa prática ocupam um espaço central na vida desse grupo de mulheres. Isso porque quase a totalidade delas reconheceu que a adesão à atividade física era condição para obterem boa disposição corporal, fortalecimento muscular, boa movimentação de ombros e braços, além de favorecer o sono, previnir estresse e ajudar na saúde mental.

Do ponto de vista da teoria de campo de Kurt Lewin, o que é importante para a investigação da dinâmica comportamental não é somente abstrair a situação em que um fenômeno ocorre, mas procurar aquelas situações em que os fatores determinantes da estrutura dinâmica total são discernidos de um modo sumamente claro, fazendo referência ao pleno concretismo das situações particulares $^{(5)}$.

Assim, no presente estudo, foi possível identificar indicadores que funcionaram como forças centrífugas, para adesão à pratica da atividade física, sendo que a falta força de vontade foi a mais apontada. Essas forças mobilizaram-nas à não-adesão da prática da atividade física de forma rotineira.

As mulheres identificaram alguns fatores determinantes para que a prática da atividade física fosse incorporada no seu espaço cotidiano como: a presença de um profissional, o suporte de familiares, o fundo musical e o ritmo, assim como a possibilidade de replicar os exercícios por meio de um manual de orientações, pois, para elas, tais indicadores se colocaram em uma posição psicológica favorável à prática da atividade física.

Nesse sentido, verificamos que o grupo de mulheres estudadas tem consciência de que a questão da adesão à prática de atividade física está ligada também a aspectos dinâmicos do cotidiano, ou seja, há necessidade de que a atividade física ocupe espaços centrais em suas vidas.

Os motivos para a prática dessa atividade estão relacionados à percepção de suceptibilidade, como o medo de desenvolver o linfedema, a própria necessidade de melhorar os movimentos dos braços e ombros, a circulação linfática.

\section{CONCLUSÕES}

Podemos concluir que, para o grupo estudado, os benefícios percebidos sobre a prática da atividade física foram melhora na disposição, ânimo, energia e bem-estar; ela é benéfica para as articulações; melhora os movimentos dos braços e ombros, fortalece os músculos, previne o linfedema e ajuda na saúde mental, ajuda a dormir melhor, e dá prazer.

A falta de força de vontade foi a barreira mais mencionada pelas mulheres.

O conhecimento sobre a importância e a necessidade dos exercícios físicos com a presença de um profissional e o suporte dos familiares, foi o incentivo mais referido pelas mulheres estudadas.

Os achados deste estudo vêm reforçar a necessidade de os profissionais de saúde que trabalham com mulheres com câncer de mama buscarem estratégias que as motivem à prática da atividade física, especialmente aquelas atividades relacionadas à prevenção, profilaxia do linfedema e mobilização dos braços e ombros.

Os resultados deste estudo revelam que a integralidade da assistência não é somente o acesso às tecnologias, chamando a atenção dos profissionais para aspectos biológicos, físicos e sociais do adoecer, que também podem traduzir a integralidade. Mostram a importância de uma equipe multidisciplinar na orientação e apoio psicológico de pacientes oncológicos onde a enfermagem tem um importante papel como facilitadora do processo de reabilitação. 


\section{REFERÊNCIAS BIBLIOGRÁFICAS}

1. Araujo RZ. Reabilitação pós mastectomia. In: Piato S. Diagnóstico e terapêutica em mastologia. $2^{\text {a }}$ ed. Rio de Janeiro (RJ): Atheneu; 1998. p.181-7.

2. Mamede MV. Reabilitação de Mastectomizadas: um novo enfoque assistencial. [Tese]. Ribeirão Preto (SP): Escola de Enfermagem de Ribeirão Preto/USP; 1991.

3. Camargo MC, Marx AG. Reabilitação física no câncer de mama. São Paulo (SP): Rocca; 2000.

4. Meirelles MCCC. Linfedema pós cirurgia por câncer de mama: avaliação de um protocolo de tratamento. [Dissertação]. Ribeirão Preto (SP): Escola de Enfermagem Ribeirão Preto/USP; 1998.

5. Lescura Y, Mamede MV. Educação em saúde: abordagem para o enfermeiro. São Paulo (SP): Sarvier; 1990.

6. Kraus PL. Body image, decision markin, and breast cancer treatment. Cancer Nurs 1999; 6(22):421-7.

7. Humble CA. Lymphedema: incidence, pathophysiology, managent, and nursing care. Continuing Educ 1995; 22(10):1503-9.

8. Ministério da Saúde (BR). Programa de educação e saúde através do exercício físico e do esporte [online]. Disponível em: URL: http://www.saude.gov.br/programas/física/ física.htm.

9. Oliveria SA, Christos PJ. The epidemiology of physical activity and cancer. Ann NY Acad Sci 1997;.833: 79-90.

10. Young DR, King AC. Preventive health issues: the mature years (64 and olders). In: Allen KM, Phillips JM. Women's Health Across the lifespan: a comprehensive perspective. Philadelphia: Lippincott; 1996. p.102-21.

11. Schwartz AL. Patterns of exercise and fatigue in physically active cancer suvivors. Oncol Nurs Forum 1998; 25(3):48591.

12. Sasaki T, Lamari NM. Reabilitação funcional precoce pós mastectomia. HB Cientíca 1997; 4(2):121-7.

13. Courneya KS, Fridenreich CM. Utility of the Theory of planed behavior for understanding exercise during breast cancer treatment. Psycho-oncology 1999; 8:112-22.

14. Erickson VS, Pearson ML, Ganz PA, Adams J, Kahn KL. Arm edema in breast cancer patients. J Natl Cancer Inst 2001; 2(93):96-111.

15. Panobianco MS. O significado do linfedema para mulheres com câncer de mama. [Tese]. Ribeirão Preto (SP): Escola de Enfermagem Ribeirão Preto/USP; 2002.

16. Graydon JE, Bubela, N, Irvine D, Vincent L. Fatiguereducing strategies used by patients receiving treatment for cancer. Cancer Nurs 1995; 18(1):23-8.

17. Leddy SK. Incentives and barriers to exercise in women with a history of breast cancer. Oncol Nurs Forum 1997; 24(5):885-90.

18. Biffi RG. A dinâmica familiar de um grupo de mulheres com câncer de mama. [Tese]. Ribeirão Preto (SP): Escola de Enfermagem Ribeirão Preto/USP; 2003. 